\title{
Chave interativa para a identificação das espécies da Aliança Tabebuia (Bignoniaceae) no estado da Bahia, Brasil
}

\author{
Fabio da Silva do Espírito Santo ${ }^{1,3}$, Alisson Amorim Siqueira ${ }^{2}$ \& Alessandro Rapini $^{1}$ \\ ${ }^{1}$ Departamento de Ciências Biológicas, Universidade Estadual de Feira de Santana - UEFS, Av. \\ Transnordestina, s/n, Novo Horizonte, CEP 44036-900, Feira de Santana, BA, Brasil. www.uefs.br/ppgbot \\ ${ }^{2}$ Universidade Federal do Vale do São Francisco - UNIVASF, Av. Antônio Carlos Magalhães, 510, Santo \\ Antônio, CEP 48902-300, Juazeiro, BA, Brasil. www.univasf.edu.br/ cpgea \\ ${ }^{3}$ Autor para correspondência: Fabio da Silva do Espírito Santo, e-mail: fse.santo@yahoo.com.br
}

ESPÍRITO SANTO, F.S., SIQUEIRA, A.A. \& RAPINI, A. Interactive key for identification of species of Tabebuia Alliance (Bignoniaceae) in the state of Bahia, Brazil. Biota Neotrop. 13(3): http://www. biotaneotropica.org.br/v13n3/en/abstract?identification-key+bn03213032013

Abstract: An interactive key to identify the 26 species of the Tabebuia Alliance (Bignoniaceae) from the state of Bahia, Brazil, was developed. From a web page containing check boxes with botanical characters, with free and not sequential marking, species which not match to the criteria are eliminated until the complete identification of the material. The key is hosted in a website, that also provides general information about the Bignoniaceae, the Tabebuia Alliance and the state of Bahia, as well as a glossary of the botanical terminology employed in the key and a bank of images. The system was created using HTML and Javascript languages. It runs from a CD-ROM and is available for download at: http://www.mendeley.com/profiles/alessandro-rapini/publications/ ComputerProgram/. The use of interactive keys facilitates and streamlines the process of taxa identification, contributing to the dissemination of biological knowledge and preparation of programs to the recognition and conservation of biota.

Keywords: multi-acess key, ipê, taxonomy, information technology.

ESPÍRITO SANTO, F.S., SIQUEIRA, A.A. \& RAPINI, A. Chave interativa para a identificação das espécies da Aliança Tabebuia (Bignoniaceae) no estado da Bahia, Brasil. Biota Neotrop. 13(3): http://www.biotaneotropica. org.br/v13n3/pt/abstract?identification-key+bn03213032013

Resumo: Foi elaborada uma chave interativa para a identificação das 26 espécies da Aliança Tabebuia (Bignoniaceae) nativas do estado da Bahia, Brasil. A partir de uma página web, contendo caixas de seleção com caracteres botânicos, de marcação livre e não sequencial, vão se eliminando as espécies que não atendem aos critérios selecionados até a identificação completa do material. A chave está inserida em um site, contendo informações sobre Bignoniaceae, a Aliança Tabebuia e o estado da Bahia, bem como um glossário dos termos botânicos utilizados na chave e um banco de imagens. O sistema foi desenvolvido a partir das linguagens HTML e Javascript, é autoexecutável em CD-ROM e está disponível para download em: http://www.mendeley.com/ profiles/alessandro-rapini/publications/ComputerProgram/. A utilização das chaves interativas facilita e dinamiza o processo de identificação dos táxons, contribuindo para a difusão do conhecimento biológico e elaboração de programas voltados ao reconhecimento e conservação da biota.

Palavras-chave: chave de múltiplo acesso, ipê, taxonomia, tecnologia da informação. 


\section{Introdução}

A Bahia possui aproximadamente $565.000 \mathrm{~km}^{2}$, sendo o maior estado da Região Nordeste e o quinto maior do Brasil - IBGE (Instituto... 2002). Seu território abriga uma grande variedade de fitofisionomias e uma alta diversidade de animais e plantas (Giulietti et al. 2006), sendo uma região prioritária para estudos de florística e conservação. O levantamento das Bignoniaceae do Brasil (Lohmann 2012) revelou que o estado está entre os mais diversos em número de espécies, com destaque para a Aliança Tabebuia (sensu Grose \& Olmstead 2007), um grupo exclusivamente neotropical que agrupa espécies de elevada importância ecológica, ornamental, medicinal e econômica (Gentry 1980). As espécies da Aliança são conhecidas popularmente como ipê ou pau-d'arco e uma delas, Handroanthus albus (Cham.) Mattos, é considerada a flor-símbolo do Brasil. A Bahia é um importante centro de diversidade da Aliança, já tendo sido inventariadas 26 espécies (Espírito-Santo et al. 2013), incluindo três espécies novas de Handroanthus (Espírito-Santo et al. 2012a, b).

A necessidade crescente de se conhecer a flora brasileira tem estimulado a criação de ferramentas que facilitem a identificação rápida e precisa de suas espécies, e as chaves de identificação têm sido amplamente utilizadas neste sentido. De acordo com Gordh \& Headrick (2001), essas chaves são dispositivos taxonômicos por meio dos quais objetos podem ser identificados com base em um conjunto de estados de caracteres. Existem diferentes tipos de chaves: pictóricas, dicotômicas e interativas; as duas primeiras são consideradas convencionais e as mais difundidas (Seltmann 2004, Brach \& Song 2005, Fujihara 2008). Para os especialistas, elas são instrumentos corriqueiros de trabalho e auxiliam na identificação das espécies. No entanto, a utilização de termos específicos e descrições incompletas ou subjetivas muitas vezes tornam essas chaves convencionais praticamente indecifráveis para aqueles menos familiarizados com o grupo em questão (Edwards \& Morse 1995, Walter \& Winterton 2007).

Diversos têm sido os esforços destinados à otimização das atividades de identificação das espécies. As ferramentas computacionais, por exemplo, vêm sendo utilizadas desde a década de 1960 (Goodall 1968, Morse 1968). As chaves interativas ou de múltiplos acessos, desenvolvidas a partir de programas computacionais, podem atrelar ferramentas adicionais, como banco de imagens, glossários e informações sobre os táxons. Nelas, não existe uma ordem pré-estabelecida para se iniciar a identificação; o usuário pode optar pelos caracteres mais visíveis dentre aqueles disponíveis no espécime. Essa flexibilidade torna a atividade menos complexa (Dallwitz et al. 2000, Walter \& Winterton 2007, Bittrich et al. 2012) e aumenta consideravelmente as chances de identificação correta, mesmo com material incompleto. Dessa maneira, as chaves interativas podem contribuir substancialmente para a popularização da taxonomia e o avanço dos estudos florísticos, sistemáticos e de conservação (Dallwitz 1980, Jarvie \& Stevens 1998, Dallwitz et al. 2000, Heidorn 2001, Silva et al. 2011, Bittrich et al. 2012).

O presente trabalho apresenta uma chave interativa para a identificação das espécies da Aliança Tabebuia nativas do estado da Bahia. A chave foi desenvolvida para atender uma ampla faixa da sociedade que se interessa pela flora brasileira, mas suas informações estão mais acessíveis àqueles com conhecimentos básicos de botânica, como técnicos e gestores ambientais, estudantes de biologia e paisagistas.

\section{Material e Métodos}

Os caracteres morfológicos foram obtidos a partir da análise de aproximadamente 1.200 exsicatas depositadas nos herbários ALCB,
BAH, BHCB, CEN, CEPEC, HRB, HST, HUEFS, HUESB, HUESC, IBGE, IPA, MBM, R, RB, SP, SPF, UB e UFP [siglas de acordo com Thiers (2013)]. Buscou-se incluir no banco de dados os caracteres vegetativos e reprodutivos que mais contribuíram para a diferenciação das espécies (Espírito-Santo et al. 2013). A terminologia morfológica está de acordo com Radford et al. (1974) e Harris \& Harris (1994). As fotografias do banco de imagens foram obtidas no campo, durante expedições realizadas entre fevereiro de 2010 e dezembro de 2012.

A construção da chave interativa teve início com a seleção e organização das características das espécies em uma planilha. $\mathrm{O}$ conjunto de valores possíveis para cada caráter foi incluído em uma lista múltipla (Figura 1). Cada elemento da lista foi identificado por um rótulo (legenda) e um valor informando se a opção é um "blocopai”, que pode ser expandida e apresentar diversas escolhas, ou um "bloco-filho", que possui apenas uma escolha. A representação interna dos dados foi obtida computacionalmente por meio de estruturas presentes na linguagem Javascript, que permitem às páginas da internet capturarem e processarem os dados contidos na interface dentro do próprio navegador, tornando a exibição mais dinâmica, e sem a necessidade de comunicação com um servidor (Flanagan 2004). Utilizou-se a linguagem HTML para exibir graficamente os dados por meio de links que permitem aos usuários a escolha dos valores de cada caráter (Figura 2).

A interface gerada possibilita a comparação dos caracteres selecionados com a lista de características de cada espécie, armazenada no formato Javascript. O algoritmo responsável pela lista de espécies possíveis com as características selecionadas é controlado pelo pseudocódigo:

\section{PARA [cada característica selecionada] FAÇA}

\section{SE (a espécie POSSUI a característica selecionada) ENTÃO}

DEIXE a espécie na lista e PASSE para a próxima espécie

\section{SENÃO}

RETIRE a espécie da lista e PASSE para a próxima espécie

\section{FIM}

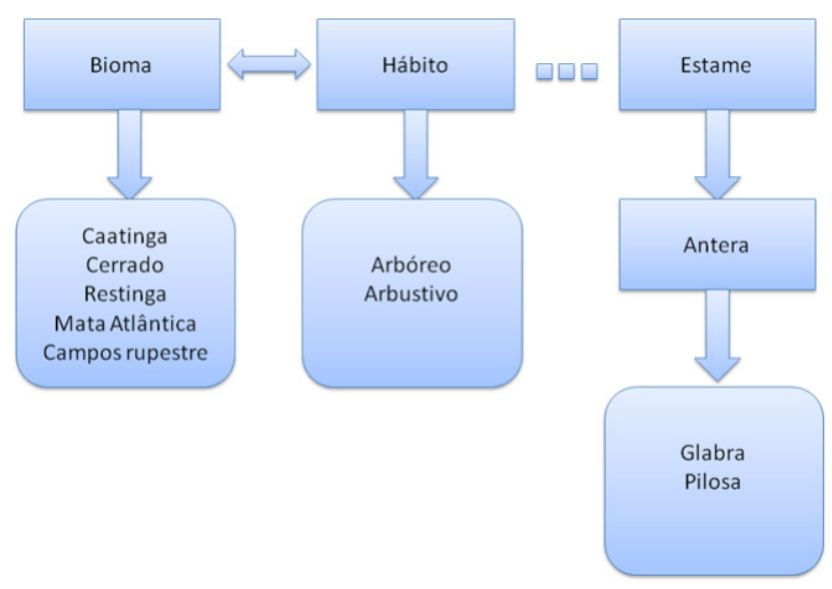

Figura 1. Diagrama ilustrando a hierarquia dos elementos utilizados na chave interativa. Cada "bloco-pai" (Bioma, Hábito ou Estame) possui um ou mais "blocos-filho" (Caatinga, Arbóreo, Antera, etc.), que podem gerar "filhos", tornando-se "pai" (Antera).

Figure 1. Diagram illustrating hierarchy of components used in the interactive key. Each main component (Biome, Habit or Stamen) has one or more derived elements (Caatinga, Tree, Anther, etc.), which may also have a derived component, in this case acting as a main component as well (Anther). 


\begin{tabular}{|c|c|}
\hline 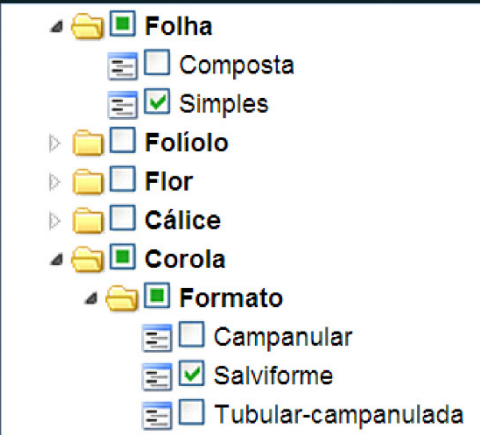 & \\
\hline Espécies Possiveis (1) & Características Selecionadas \\
\hline Tabebuia stenocalyx & $\begin{array}{l}\text { [Folhas]: Simples } \\
\text { [Corola][Formato]: Salviforme }\end{array}$ \\
\hline
\end{tabular}

Figura 2. Interface gráfica da chave interativa cruzando as características selecionadas com o banco de espécies.

Figure 2. Graphic interface of the interactive key matching selected characters with species list.

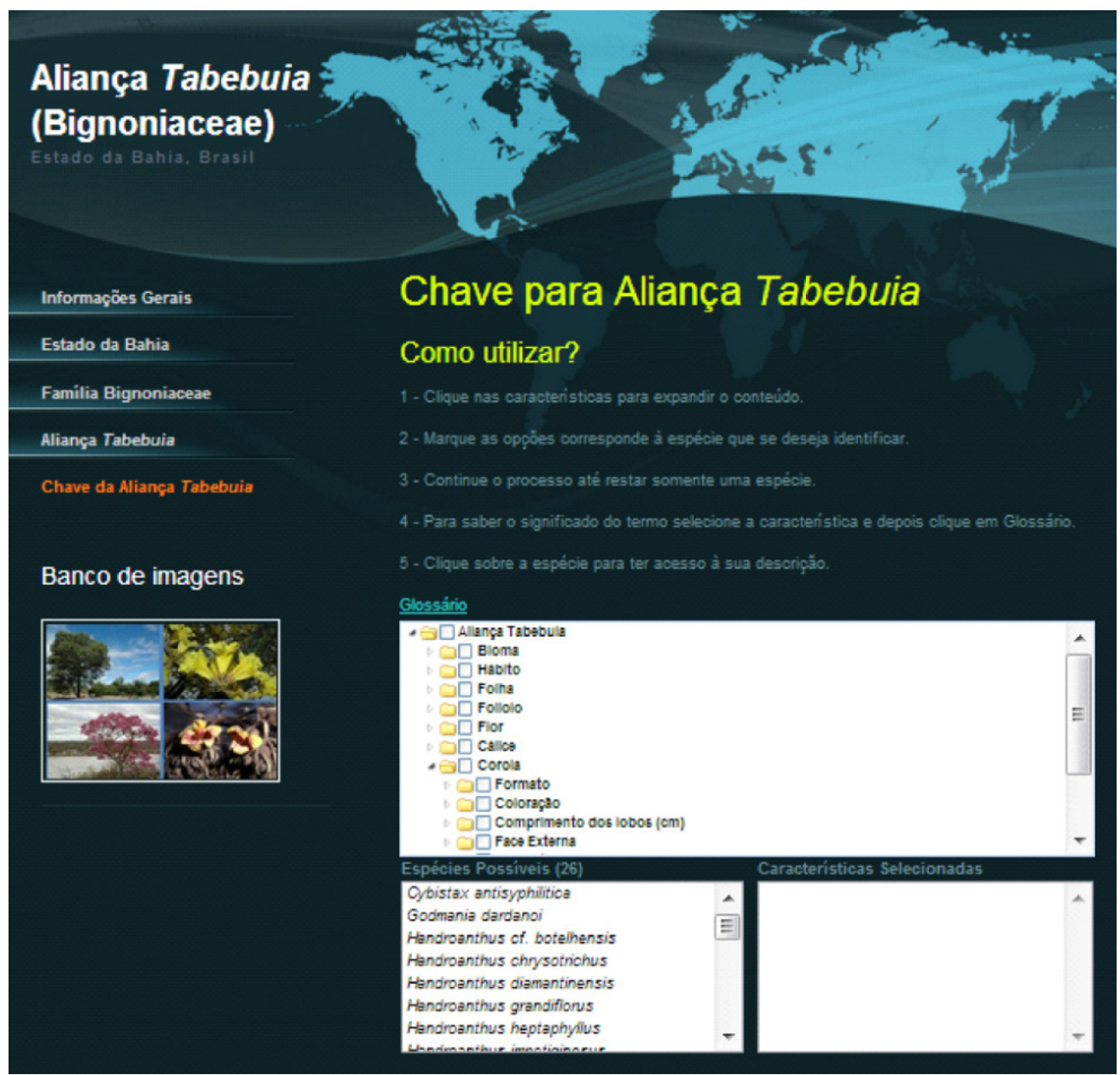

Figura 3. Interface gráfica do site evidenciando, no menu esquerdo, as informações atreladas à chave, e à direita, informações de como ela deve ser utilizada, o glossário e os três blocos funcionais: campo com as características que podem ser selecionadas, bloco com as espécies possíveis e outro onde as características ficam armazenadas.

Figure 3. Graphic interface of the website showing information regarding the key in the left menu, and at right, information on how it should be used, the glossary and the three functional blocks: field with the features that can be selected, the block with possible species and another one where the features are stored. 
Não existe uma ordem específica para checagem das características e o número de espécies tende a diminuir a cada item selecionado, até que reste apenas uma espécie (Figura 2). O código e a interface para a escolha das características foram inseridos em uma página web, tornando a chave interativa acessível pela internet.

\section{Resultados}

A interface do site, disponível para download em http:// www.mendeley.com/profiles/alessandro-rapini/publications/ ComputerProgram/, é composta por um menu com cinco links (Figura 3), onde são apresentadas informações gerais sobre o projeto, a área de estudo, a família Bignoniaceae e a Aliança Tabebuia. Neste item, também estão inseridos a chave de identificação interativa, um glossário botânico com os termos utilizados na chave e um banco de imagens com 72 fotografias.

Foram analisados e incluídos na chave 33 caracteres morfológicos, além de informações sobre o bioma onde ocorrem, descrição e comentários gerais sobre a distribuição e fenologia reprodutiva de todas as espécies. No site também podem ser encontradas instruções para o uso da chave. A chave é composta por três blocos funcionais: o primeiro inclui as informações referentes às espécies (biomas em que ocorrem, hábito e informações morfológicas das folhas, flores, frutos e sementes), o segundo é a lista das 26 espécies que ocorrem no estado (espécies possíveis) e o terceiro armazena as características selecionadas (Figura 3). A cor da corola e o formato da cápsula são os caracteres que mais auxiliam na diferenciação das espécies em grupos. Portanto, sugere-se que a identificação comece por esses caracteres. Independente da coloração selecionada, por exemplo, o número de espécies possíveis é reduzido em mais de $50 \%$. Algumas características ou combinação destas são exclusivas de determinados táxons, por exemplo: corola verde e cápsulas elipsoide-oblongas identificam Cybistax antisyphilitica; corola salviforme, Tabebuia stenocalyx; anteras pilosas e cápsulas cilíndricoespiraladas, Godmania dardanoi; lobos da corola maiores que 3,0 cm de comprimento, Handroanthus grandiflorus; ovário estipitado e cápsulas muricadas, o gênero Zeyheria.

\section{Discussão}

A possibilidade de se agregar informações adicionais às chaves interativas, como dados acerca da área e do grupo de estudo propiciam um ambiente favorável para iniciar a identificação. Inicialmente, é necessário selecionar os caracteres que podem ser examinados no material disponível. A flexibilidade de se iniciar a identificação por qualquer caráter é uma das principais vantagens das chaves com múltiplos acessos. Como os caracteres são dispostos de forma isolada e independente, a falta de uma estrutura da planta geralmente não compromete sua identificação. No entanto, a aplicação combinada de caracteres (e.g., folha, cálice e corola) propicia uma identificação mais rápida e precisa. Em algumas situações, especialmente para espécimes estéreis ou apenas com fruto, o processo pode indicar mais de uma espécie possível. Neste caso, recomenda-se analisar as descrições dessas espécies (clicando sobre o nome), além de consultar o banco de imagens para se concluir a identificação do material.

Apesar da versatilidade das chaves com múltiplos acessos, este recurso ainda é pouco utilizado para auxiliar na identificação das espécies brasileiras. Um exemplo são as chaves interativas produzidas pelo Departamento de Biologia Vegetal da UNICAMP: http://www2. ib.unicamp.br/profs/volker/chaves. Até o momento, no entanto, desconhecemos a publicação de chaves de múltiplos acessos voltadas para a identificação de espécies de plantas no estado da Bahia. A facilidade para atualização e acréscimo de novos táxons, caracteres e informações somados ao acesso rápido a partir de CD-ROM ou internet são as principais vantagens das chaves interativas quando comparadas às chaves convencionais. Tais benefícios contribuem diretamente para a difusão do conhecimento biológico e podem servir como subsídios para a elaboração de projetos e programas voltados ao reconhecimento e conservação da biota.

\section{Agradecimentos}

Agradecemos ao CNPq pela concessão de bolsa de mestrado ao primeiro autor e de produtividade (PQ-1D) ao terceiro autor; à Fapesb, pelo apoio ao projeto Flora da Bahia, e ao MCT, pelo apoio ao Programa de Pesquisa em Biodiversidade (PPBio) do Semiárido.

\section{Referências Bibliográficas}

BITTRICH, V., SOUZA, C.S.D., COELHO, R.L.G., MARTINS, M.V., HOPKINS, M.J.G. \& AMARAL, M.C.E. 2012. An interactive key (Lucid) for the identifying of the genera of seed plants from the Ducke Reserve, Manaus, AM, Brasil. Rodriguésia 63:55-64. http://dx.doi.org/10.1590/ S2175-78602012000100005

BRACH, A.R. \& SONG, H. 2005. ActKey: a web-based interactive identification key program. Taxon 54: 1041-1046. http://dx.doi. org/10.2307/25065490

DALLWITZ, M.J. 1980. A general system for coding taxonomic information. Taxon 29:41-46. http://dx.doi.org/10.2307/1219595

DALLWITZ, M.J., PAINE, T.A. \& ZURCHER, E.J. 2000. Principles of interactive keys. http://delta-intkey.com (último acesso em 17/12/2011).

EDWARDS, M. \& MORSE, D.R. 1995. The potential for computer-aided identification in biodiversity research. Trends Ecol. Evol. 10:153-158. http://dx.doi.org/10.1016/S0169-5347(00)89026-6

ESPÍRITO-SANTO, F.S., SILVA-CASTRO, M.M. \& RAPINI, A. 2012a. Handroanthus grandiflorus (Bignoniaceae), a new species from the semiarid region of Brazil. Phytotaxa 48:1-6. http://www.mapress.com/ phytotaxa/content/2012/1/pt00048p006.pdf

ESPÍRITO-SANTO, F.S., SILVA-CASTRO, M.M \& RAPINI, A. 2012b. Two new species of Handroanthus Mattos (Bignoniaceae) from the state of Bahia, Brazil. Acta Bot. Bras. 26:651-657. http://dx.doi.org/10.1590/ S0102-33062012000300014

ESPÍRITO-SANTO, F.S., SILVA-CASTRO, M.M. \& RAPINI, A. 2013. Flora da Bahia: Bignoniaceae 2 -Aliança Tabebuia. Sitientibus, ser. Ci. Biol. 13: http://pkp.uefs.br/ojs/index.php/sitientibusBiologia/article/view/211/282 (último acesso em 26/07/2013).

FLANAGAN, D. 2004. Javascript - o guia definitivo. Ed. Bookman, Porto Alegre.

FUJIHARA, R.T. 2008. Chave pictórica de identificação de famílias de insetos-pragas agrícolas. Dissertação de mestrado, Universidade Estadual Paulista, Botucatu.

GENTRY, A.H. 1980. Bignoniaceae, Part I. Tribes Crescentieae and Tourrettieae. Flora Neotropica Monograph 25:1-131.

GIULIETTI, AM., QUEIROZ, L.P., SILVA, T.R.S., FRANÇA, F., GUEDES, M.L. \& AMORIM, A.M. 2006. Flora da Bahia. Sitientibus, ser. Ci. Biol. 6(3):169-173.

GOODALL, D.W. 1968. Identification by computer. BioScience: 18:485-488. http://dx.doi.org/10.2307/1294274

GORDH, G. \& HEADRICK, D. 2001. A dictionary of entomology. CABI Publishing, Wallingford.

GROSE, S.O. \& OLMSTEAD, R.G. 2007. Evolution of a charismatic neotropical tree: molecular phylogeny of Tabebuia s. l., and allied genera (Bignoniaceae). Syst. Bot. 32:650-659. http://dx.doi. org $/ 10.1600 / 036364407782250553$

HARRIS, J.G. \& HARRIS, M.W. 1994. Plant identification terminology: an illustrated glossary. Spring Lake Publishing, Spring Lake. 
HEIDORN, P.B. 2001. A tool for multipurpose use of online flora and fauna: the biological information browsing environment (BIBE). First Monday 6(2): http://firstmonday.org/ojs/index.php/fm/article/view/835/744 (último acesso em 28/02/2013)

INSTITUTO BRASILEIRO DE GEOGRAFIA E ESTATÍSTICA - IBGE. 2002. Área territorial oficial. Resolução da presidência do IBGE de $n^{\circ} 5$, de 10 de outubro de 2002. IBGE. http://www.ibge.gov.br/home/geociencias/ areaterritorial/principal.shtm (último acesso em 15/01/2012).

JARVIE, J.K. \& STEVENS, P.F. 1998. Interactive keys, inventory, and conservation. Conserv. Biol. 12:222-224. http://dx.doi.org/10.1046/ j.1523-1739.1998.96123.x

LOHMANN, L.G. 2012. Bignoniaceae. In: Lista de espécies da flora do Brasil. Jardim Botânico do Rio de Janeiro, Rio de Janeiro. http://floradobrasil. jbrj.gov.br/jabot/floradobrasil/FB112305 (último acesso em 28/01/2012).

MORSE, L.E. 1968. Construction of identification keys by computer. Am. J. Bot. 55:737.
RADFORD, A.E., DICKINSON, W.C., MASSEY, J.R. \& BELL, C.R. 1974. Vascular plant systematics. Harper \& Row, New York.

SELTMANN, K. 2004. Building web-based interactive keys to the hymenopteran families and superfamilies. M.Sc. thesis. University of Kentucky, Lexington.

SILVA, H., PINHO, R., LOPES, L., NOGUEIRA, A.J.A. \& SILVEIRA, P. 2011. Illustrated plant identification keys: An interactive tool to learn botany. Comput. Educ. 56:969-973. http://dx.doi.org/10.1016/j. compedu.2010.11.011

THIERS, B. 2013. Index Herbariorum: a global directory of public herbaria and associated staff. New York Botanical Garden's Virtual Herbarium. http://sweetgum.nybg.org/ih (último acesso em 28/02/2013).

WALTER, D.E. \& WINTERTON, S. 2007. Keys and the crisis in taxonomy: extinction or reinvention? Annu. Rev. Entomol. 52:193-208. PMid:16913830. http://dx.doi.org/10.1146/annurev. ento.51.110104.151054 